\title{
Custom-made double inner-branched aortic arch endograft for the treatment of mycotic aortic arch aneurysm: a case report
}

\author{
Benien JP Hau, BBiomed, MB, BS, YC Chan *, MD, FRCS, Stephen W Cheng, MS, FRCS
}

Division of Vascular and Endovascular Surgery, Department of Surgery, Queen Mary Hospital, The University of Hong Kong, Hong Kong

*Corresponding author: ycchan88@hkucc.hku.hk

Hong Kong Med J 2021;27:452-5

https://doi.org/10.12809/hkmj209129

\section{Case report}

A 76-year-old frail man was admitted to hospital with fever and delirium. His medical history was significant for diabetes mellitus, hypertension, and dyslipidaemia. White blood cell count was $9.3 \times 10^{9} / \mathrm{L}$ and C-reactive protein $18.20 \mathrm{mg} / \mathrm{dL}$. Blood cultures yielded serogroup D Salmonella enteritidis. Computed tomography (CT) angiogram showed an aortic arch aneurysm of $4.7 \mathrm{~cm}$ (Fig 1a). Gallium scintiscan confirmed focal uptake at the proximal aortic arch. He developed progressively worsening chest discomfort for the subsequent 3 weeks, and a new CT angiogram showed rapid increase in the aneurysm size to $5.3 \mathrm{~cm}$ (Fig 1b).

A diagnosis of mycotic aortic arch aneurysm was made, and multidisciplinary consultation concluded that he was too frail to undergo conventional open repair. A custom-made double inner-branched aortic arch endograft (Cook Medical, Bloomington [IN], US) was arranged. During the interim, he received intravenous ceftriaxone. The left subclavian artery was revascularised with a left common carotid artery (CCA) to left subclavian artery bypass as a first-stage procedure. The custom-made endograft was available 3 weeks later (Figure 2a,b) and the endovascular procedure was performed accordingly.

Briefly, all procedures were performed under general anaesthesia in a well-equipped hybrid operating room by experienced endovascular surgeons. The endografts were flushed with heparinised saline multiple times to completely evacuate trapped gas in the sheath. Access was gained through an open groin cutdown exposing the common femoral artery and vein, and bilateral neck cutdowns to expose the right and left CCA. After full systemic heparinisation and under fluoroscopic guidance, a Terumo wire and pigtail catheter were positioned in the left ventricle and exchanged for a double-curved extended Lunderquist Extra-Stiff Wire (Cook Medical). The main stent graft body was then delivered to the aortic arch. The branched endograft was deployed under fluoroscopy, with controlled systolic hypotension by an inferior vena cava occlusion balloon (Coda $46 \mathrm{~mm}$ balloon; Cook Medical) introduced via the right femoral vein.

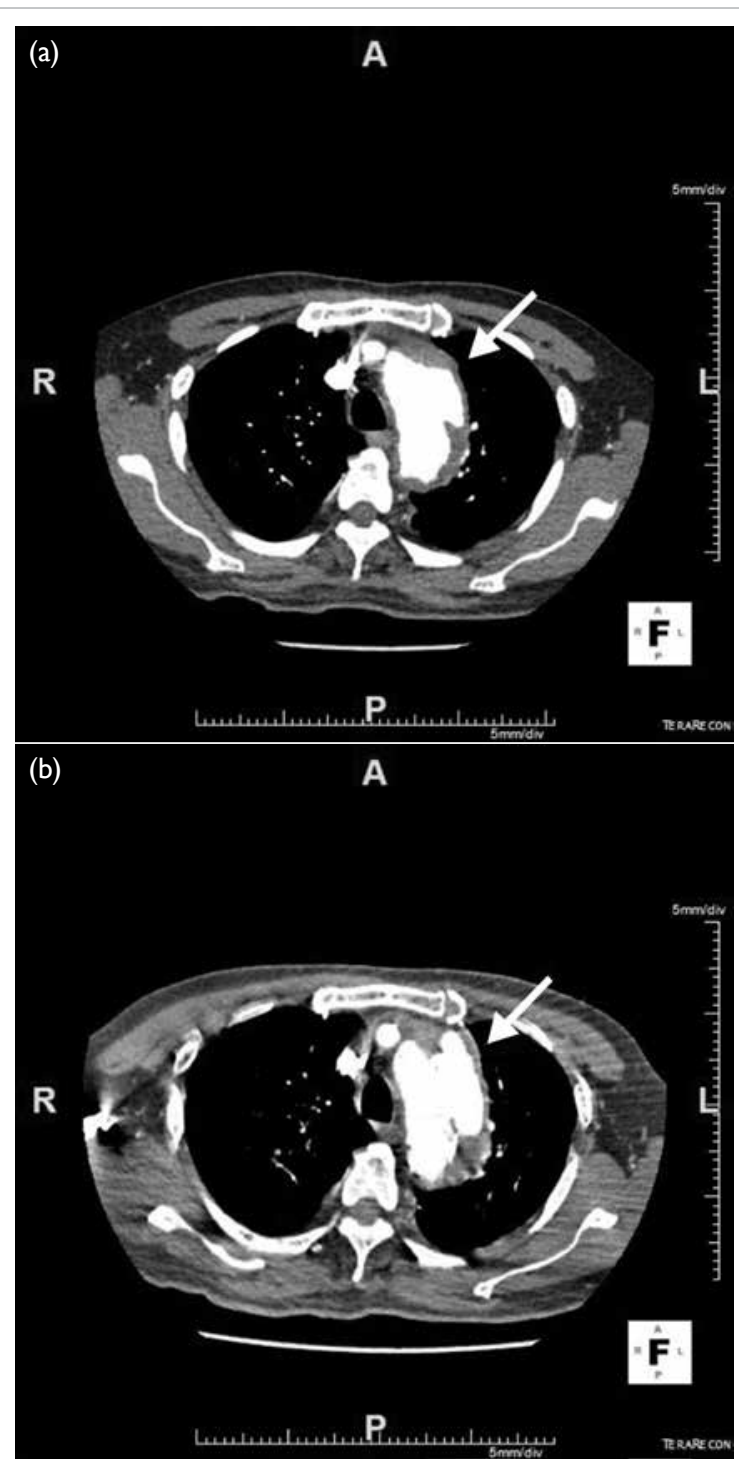

FIG I. A 76-year-old man presenting with fever and delirium. Computed tomography (CT) angiograms revealed (a) an aortic arch aneurysm of $4.7 \mathrm{~cm}$ (arrow). New CT angiogram showed (b) rapid enlargement to $5.3 \mathrm{~cm}$ after 3 weeks (arrow)

Accurate alignment of the orientation markers with the coronary and supra-aortic vessels was essential. After deployment of the main stent graft, the 


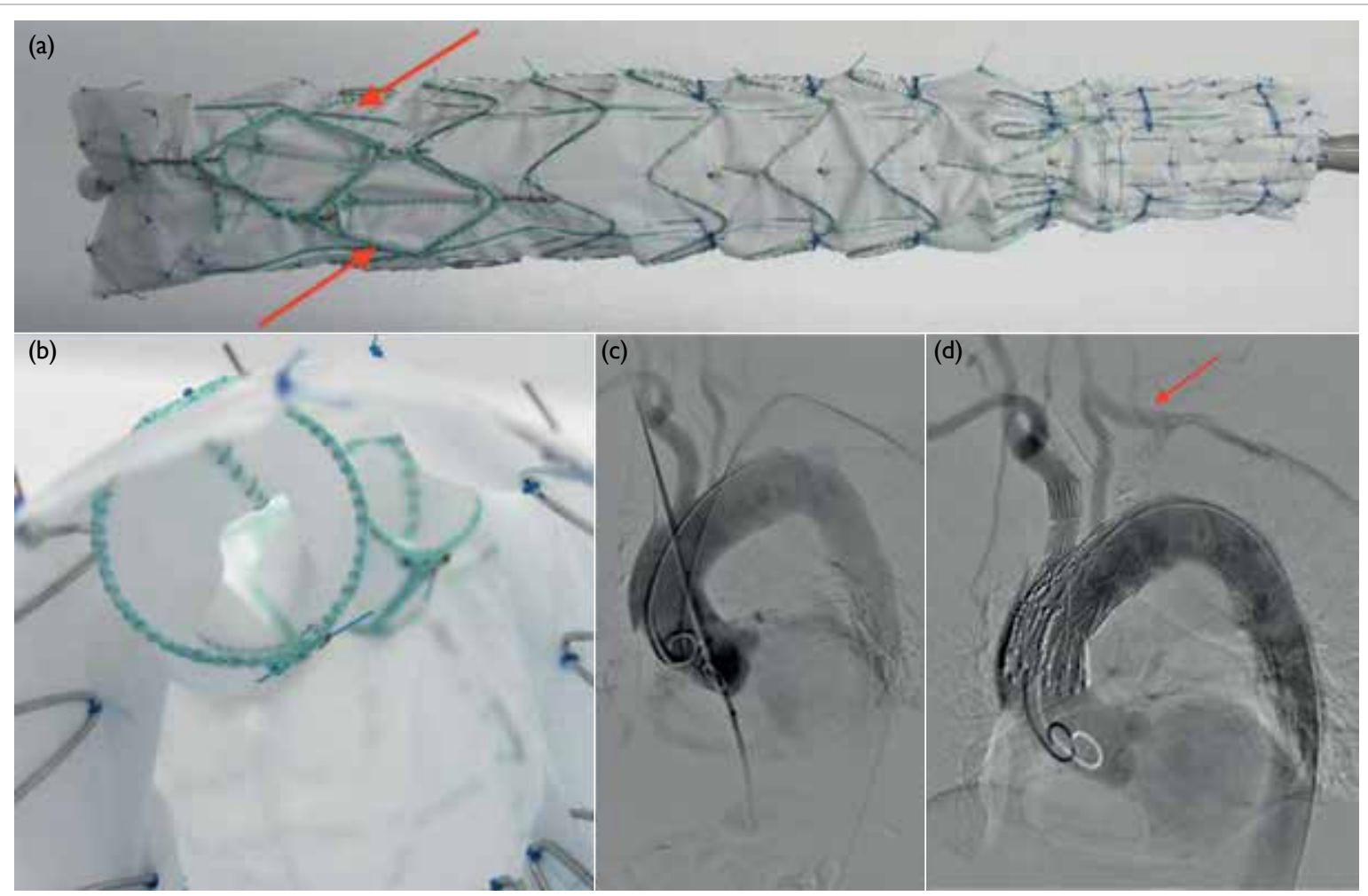

FIG 2. (a,b) Photographs of the custom-made double inner-branched aortic arch endograft (Cook Medical, Bloomington [IN], US) showing side branches at 12:30 and I I:30 (arrows). (c) Intraoperative angiogram and (d) completion angiogram showing successful exclusion of the aneurysm and preservation of all supra-aortic branches. The left subclavian and vertebral arteries are perfused by the left common carotid artery to left subclavian artery bypass graft (arrow)

proximal inner branch was sequentially cannulated in a retrograde fashion through the exposed carotid arteries. A custom-made thoracic stent graft extension was used (Cook Medical) to bridge the proximal inner branch and the innominate artery. A similar procedure was repeated for the distal inner branch via the left carotid artery, where a Fluency self-expanding covered stent (CR Bard, Murray Hill [NJ], US) was used to bridge the distal inner branch and the left CCA, taking care not to cover the left CCA to left subclavian artery bypass. The left subclavian artery origin was finally occluded with an Amplatzer vascular plug (St Jude Medical, Saint Paul [MN], US) to prevent endoleak into the sac. Completion angiogram showed successful exclusion of the aneurysm with patent supra-aortic branches (Fig 2c,d).

The patient recovered well without neurological sequelae. He was discharged on postoperative day 13 with lifelong oral ciprofloxacin prescribed. Computed tomography angiogram at 3 months (Fig 3a,b) and 1 year (Fig 3c,d) after surgery showed successful and durable results.

\section{Discussion}

To the best of our knowledge, this is the first reported use of a custom-made double innerbranched thoracic aortic endograft for treatment of Salmonella-related mycotic aortic arch aneurysm. In septic and frail patients, a custom-made endovascular device should not generally be first-line treatment as the manufacturing time may take up to a few months. However, in our patient, urgency was emphasised and availability of the stent-graft expedited.

Conventional open repair has always been the gold standard therapy for mycotic aortic aneurysms, but endovascular stent grafts may be a temporising or permanent option in critically ill patients who will not tolerate open surgery. ${ }^{1,2}$ Custom-made branched thoracic endovascular aortic repair now adds to the armamentarium of options. The use of custom-made inner-branched thoracic endografts is technically challenging and requires endovascular expertise and experience. Meticulous preoperative planning with analysis using the iNtuition workstation (TeraRecon, San Mateo [CA], US) is paramount, with reference to the characteristics of the proximal and distal landing zones in relation to the diameter, angulation, and length of the supra-aortic arteries. The cervical left CCA to left subclavian artery bypass debranching procedure can be a staged or simultaneous procedure. The operative technique is well described in published literature. ${ }^{3,4}$ 


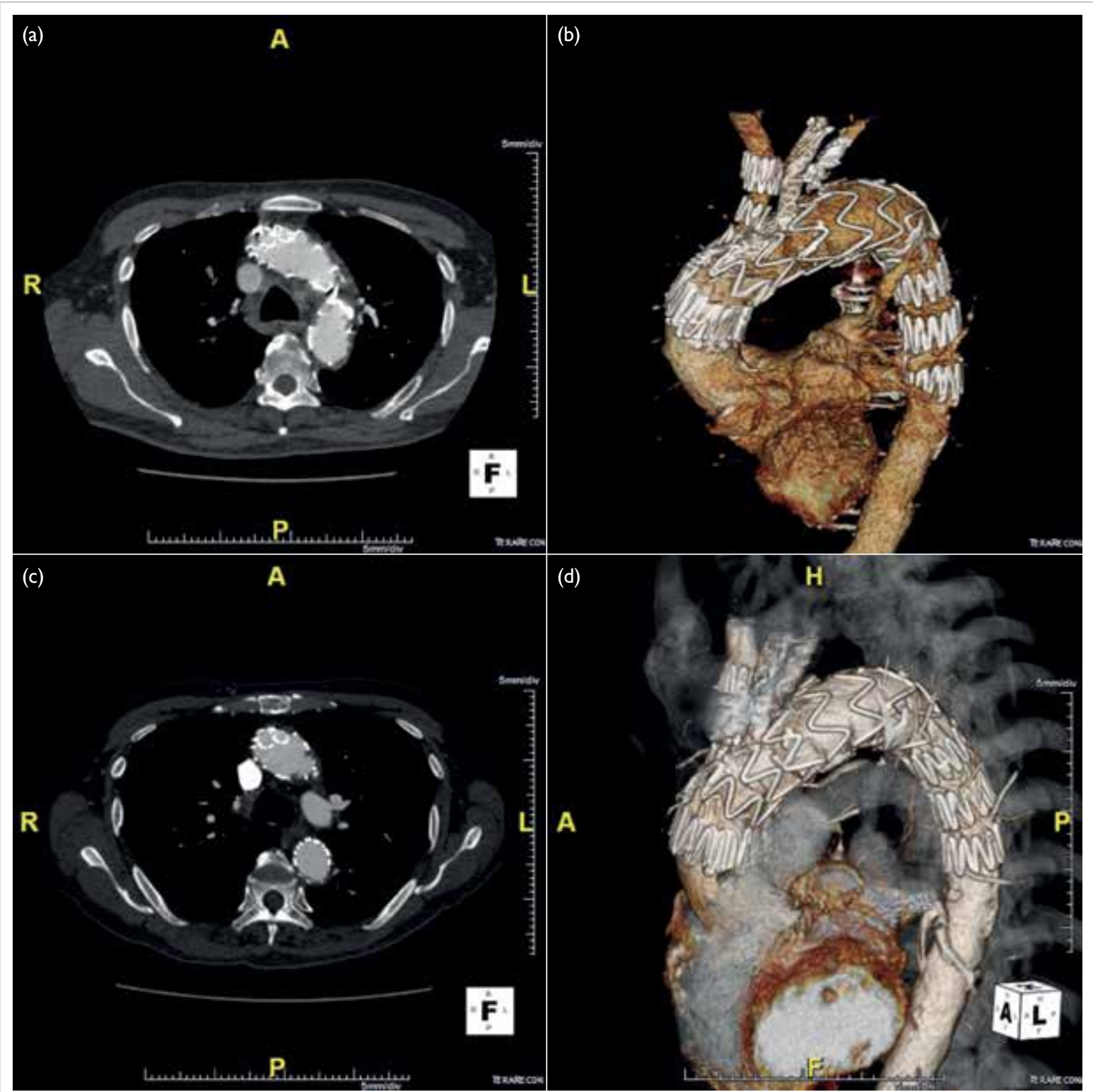

FIG 3. Computed tomography angiograms taken (a, b) 3 months and (c, d) I year after endovascular repair showing successful and durable treatment with shrinkage of the aneurysm

As with most endovascular aortic arch repairs, the most feared complication is of stroke that can occur when atherosclerotic or gaseous emboli are released from the arch or proximal supra-aortic branches during wire and graft manipulation, from clamping of carotid arteries, or due to perioperative fluctuation of blood pressure. Another major concern after stenting the ascending aorta is the risk of retrograde type A dissection, which can be mitigated by controlled hypotension during the deployment of the stent graft. Care must also be taken to not cover the orifices of the coronary arteries with the stent graft.

Off-the-shelf solutions for single and double inner-branched aortic arch endografts are currently under development and were not available at the time of preparation of this case report. The use of a custom-made thoracic endograft is a major development for these patients who would otherwise require traditional open repair, frozen elephant trunk procedure, or hybrid procedures. Frail elderly patients with co-morbidities or prior sternotomies would be denied surgery as they would not tolerate extracorporeal cardiopulmonary bypass with deep hypothermic circulatory arrest. Endovascular experience and technical support are important as the access vessel is remote to the arch. There is often a fine balance between the procedure and maintenance of cerebral perfusion. Comparing non-custom chimney and custom graft for arch pathology, O'Callaghan et $\mathrm{al}^{5}$ showed that mortality was higher in the non-custom group (7\% vs $18 \%$ ), 
and a trend favouring better durability of fenestrated grafts for sealing and re-intervention rates was noted. All patients should have regular $\mathrm{CT}$ surveillance to monitor durability and exclude aneurysm-related complications. Long-term postoperative antibiotic therapy is also important since endovascular options preclude debridement of infected tissue.

This new technique involving a custom-made double inner-branched aortic arch endograft can be considered in patients with mycotic aneurysms of the thoracic arch, with favourable and durable results.

\section{Author contributions}

All authors contributed to the concept or design of the study, acquisition of the data, analysis or interpretation of the data, drafting of the manuscript, and critical revision of the manuscript for important intellectual content. All authors had full access to the data, contributed to the study, approved the final version for publication, and take responsibility for its accuracy and integrity.

\section{Conflicts of interest}

All authors have disclosed no conflicts of interest.

\section{Funding/support}

This study received no specific grant from any funding agency in the public, commercial, or not-for-profit sectors.

\section{Ethics approval}

The patient was treated in accordance with the Declaration of Helsinki. The patient provided written informed consent for all procedures.

\section{References}

1. Chan YC, Morales JP, Taylor PR. The management of mycotic aortic aneurysms: is there a role for endoluminal treatment? Acta Chir Belg 2005;105:580-7.

2. Taylor PR, Chan YC. Endovascular treatment in the management of mycotic aortic aneurysms. In: Thompson MM, Morgan RA, Matsumura JS, Sapoval M, Loftus IM, editors. Endovascular Intervention for Vascular Disease. Principles and Practice. Boca Raton: Taylor \& Francis Group; 2008: 235-42.

3. Fiorucci B, Tsilimparis N, Rohlffs F, Heidemann F, Debus ES, Kölbel T. How to confirm catheterization of inner branches in aortic endografting: The Universal Flush Test. J Endovasc Ther 2017;24:539-41.

4. Tsilimparis N, Detter C, Law Y, et al. Single-center experience with an inner branched arch endograft. J Vasc Surg 2019;69:977-85.e1.

5. O'Callaghan A, Mastracci TM, Greenberg RK, Eagleton MJ, Bena J, Kuramochi Y. Outcomes for supra-aortic branch vessel stenting in the treatment of thoracic aortic disease. J Vasc Surg 2014;60:914-20.

\section{Answers to CME Programme Hong Kong Medical Journal October 2021 issue}

Hong Kong Med J 2021;27:338-49
I. Multicentre study of hospitalised patients with sports- and recreational cycling- related traumatic brain injury in Hong Kong
A
1. True
2. False
3. True
4. False
5. True
B
1. True
2. True
3. True
4. False
5. False

Hong Kong Med J 2021;27:350-4

\section{Total knee arthroplasty is safe for patients aged $\geq 80$ years in Hong Kong}
A
1. False
2. True
3. True
4. False
5. False
B
1. False
2. True
3. False
4. False
5. True 